CLINICAL STUDY

\title{
Associations of remission status and lanreotide treatment with quality of life in patients with treated acromegaly
}

\author{
Shih-Che Hua, Yuan-Horng Yan and Tien-Chun Chang ${ }^{1}$ \\ Division of Endocrinology and Metabolism, Department of Internal Medicine, Chia-Yi Christian Hospital, No. 539, Jhongsiao Road, Chiayi City 60002, \\ Taiwan and ${ }^{1}$ Division of Endocrinology and Metabolism, Department of Internal Medicine, National Taiwan University Hospital, College of Medicine, \\ National Taiwan University, No. 7, Chung-Shan South Road, Taipei 10002, Taiwan
}

(Correspondence should be addressed to T-C Chang; Email: tcchang@ha.mc.ntu.edu.tw)

\begin{abstract}
Objective: Acromegaly has an important impact on quality of life (QOL). The aim of this study was to evaluate the associations of remission status and lanreotide treatment with QOL in patients with treated acromegaly, by the newly developed disease-generated acromegaly QOL questionnaire (ACROQOL).

Design: Cross-sectional study.

Methods: Fifty-two patients with treated acromegaly were recruited to complete the Chinese version of the ACROQOL translated and validated from the English version. These patients were divided into controlled and uncontrolled groups based on the latest remission criteria and further subdivided into four groups according to the present treatment with lanreotide or not. Comparisons between groups were analyzed.

Results: There was no difference between controlled and uncontrolled groups in the ACROQOL scores of total score, both scales and psychological subscales. However, in the controlled group, present treatment with lanreotide, in comparison with no treatment, showed worse ACROQOL scores in total score $(P=0.021)$, psychological scale $(P=0.011)$, psychological subscale 'appearance' $(P=0.032)$ and 'personal relations' $(P=0.010)$.

Conclusions: The lanreotide treatment was negatively associated with QOL in biochemically controlled acromegalic patients, especially in the psychological aspect.
\end{abstract}

European Journal of Endocrinology 155 831-837

\section{Introduction}

Acromegaly is a rare disorder with an annual incidence of 3-4 cases per million and a prevalence of 50-70 cases per million population, being more common in women and mostly caused by a growth hormone (GH)secreting pituitary adenoma (1-2). Mortality in acromegaly is at least twice that of the general population, mostly related to cardiovascular diseases and malignancies (3-8). One large retrospective cohort study demonstrated that post-treatment GH levels $<2.5 \mu \mathrm{g} / \mathrm{l}$ $(116.25 \mathrm{pmol} / \mathrm{l})$ result in an overall mortality rate similar to that of the general population (4). The most recent broadly accepted definition of biochemical cure of acromegaly was based on the Internal Consensus Conference of Cortina d'Ampezzo in 1999 (9). Aggressive management is mandatory to achieve biochemical disease control and thereby restore life expectancy to normal. Present treatment modalities include transsphenoidal surgery, radiotherapy and medical therapy (dopamine agonists, somatostatin analogues and GH receptor antagonist).
Recently, besides the biochemical variables, clinicians have started to focus on the health-related quality of life (HR-QOL) as the outcome measurement of acromegaly and its treatment. The acromegaly quality of life questionnaire (ACROQOL), initially developed in Spain and first published in 2002, is the first acromegaly disease-generated quality of life questionnaires to specifically assess dimensions of HR-QOL most likely to be affected in patients with acromegaly (10-13). The original Spanish questionnaire was first translated into English following recommended standard methodology $(10,14)$.

Several studies have demonstrated that administration of somatostatin analogues ameliorates the abnormal biochemical profiles and the associated somatic impairments (15-19). However, whether these improvements translate into improved physical function remains largely unknown, and their impact on HR-QOL is still controversial (20). To date, there is paucity of data even attempting to correlate disease modification endpoints with quality of life in acromegaly. 
The present study was aimed to evaluate the associations of remission status and slow-release somatostatin analogue (lanreotide) treatment with HR-QOL in patients with treated acromegaly using the Chinese version of ACROQOL.

\section{Subjects and methods}

\section{Study protocol}

Using a cross-sectional design, we prospectively recruited the patients with treated acromegaly between September 2005 and January 2006 at the Division of Endocrinology and Metabolism of the National Taiwan University Hospital, Taipei, Taiwan. At first, we translated the validated English version of ACROQOL into Chinese following recommended standard methodology $(10,14)$. The English version of ACROQOL is shown in Figure 1, comprising 22 questions covering physical ( 8 items) and psychological aspects (14 items), which is further divided into two subscales: one assesses appearance-related aspects (7 items) and the other measures the impact of disease on personal relationships (7 items).

The English version was translated into Chinese by two professional, bilingual translators with lots of experience in translating HR-QOL questionnaires. Both translations were compared with each other and with the original English version at a consensus meeting consisting of endocrinologists specifying in acromegaly. If the translation was clear and correct, no changes were made. If there were doubts or contrasting opinions with the project director, a consensus was reached after extensive discussion, to produce the first Chinese version of the questionnaire. This version was then independently translated back into English to ascertain equivalent significance in both the languages. After the second meeting, the second consensus Chinese version was produced, and presented to three Chinesespeaking Taiwanese patients with acromegaly to assess and correct for clarity, cultural relevance, comprehension and suitable wording, thus providing the final Chinese version of the ACROQOL questionnaire.

The study project was approved by the Institutional Review Board of the National Taiwan University Hospital. Patients were asked to answer the 22 items

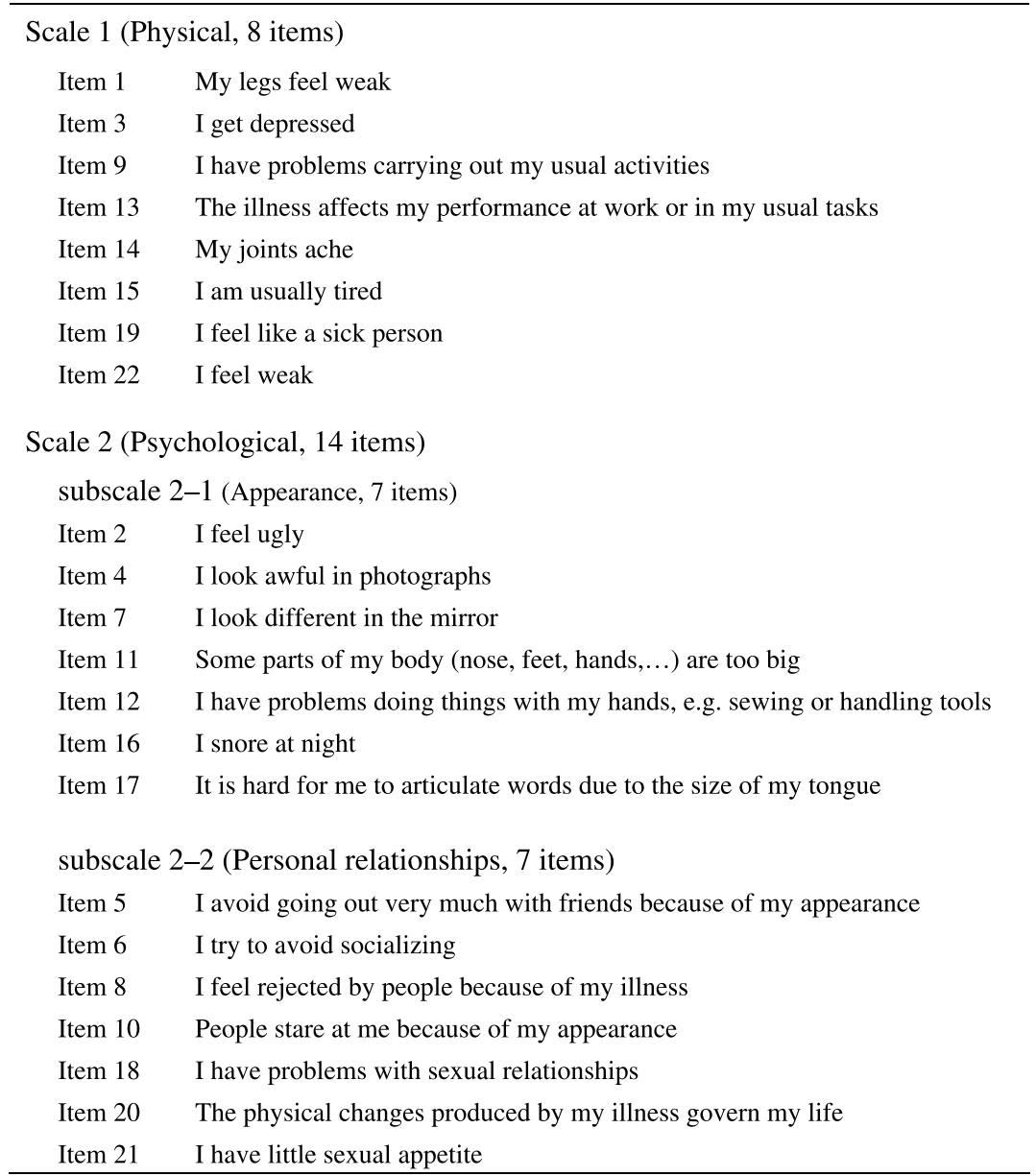

Figure 1 The acromegaly quality of life questionnaire (ACROQOL). 
of the Chinese version of the ACROQOL either by telephone visit or during their regular clinical visit. Out of 52 patients, 47 completed the questionnaire in our outpatient clinic and 4 by phone because they were treated in another hospital. All were conducted by the same researcher (S-C Hua) and in the same fashion.

The individual clinical variables, including age, gender, age of definite diagnosis, macro- or microadenoma, operation history, repeat operation history, radiotherapy history, the previous and the present treatment modalities, latest $\mathrm{GH} /$ insulin-like growth factor-I (IGF-I) levels, whether on hormone replacement therapy, and morbidities related to acromegaly were recorded by chart review. Those treated by slow-release somatostatin analogues were all using lanreotide every 14 days. For the four patients treated in another hospital, we obtained the clinical variables provided by the patients themselves. Two of them had markedly elevated GH/IGF-I levels and another two had oral glucose tolerance test (OGTT) in our outpatient clinic for confirmation of suppressible $\mathrm{GH}$ level.

The study was performed according to the declaration of Helsinki. Each patient gave informed consent personally or by mail.

\section{Assessment of biochemically controlled (remission) status}

The remission criteria were defined according to the Internal Consensus Conference of Cortina d'Ampezzo in 1999 (9) as follows: normal age- and gender-matched IGF-I concentration, basal GH level less than $2.5 \mu \mathrm{g} / \mathrm{l}$ (116.25 pmol/l) and suppression of $\mathrm{GH}$ to $<1 \mu \mathrm{g} / \mathrm{l}$ (46.5 pmol/l) during OGTT.

\section{Grouping}

The enrolled patients were divided into controlled and uncontrolled groups based on the remission criteria defined by the Internal Consensus Conference of Cortina d'Ampezzo in 1999. The controlled and the uncontrolled groups were further subdivided into with or without present lanreotide treatment groups.

\section{Tumour classification}

Pituitary adenomas were classified by the size on the magnetic resonance imaging, computed tomography or intra-operative findings: tumour diameter of $<1 \mathrm{~cm}$ was defined as microadenoma. Those $\geq 1 \mathrm{~cm}$ were defined as macroadenoma.

\section{Hormone replacement}

Pituitary function of each patient was periodically evaluated. Thyroid deficiency was defined as low free thyroxine (T4) level. Adrenal insufficiency was defined as low cortisol level with requirement of glucocorticoids supplementation and failure to be tapered off. Gonadal insufficiency was defined as low testosterone (male) or oestrogen/progesterone (female) levels.

\section{Scoring of the ACROQOL}

Each of the 22 items was answered in a 1 (worst) to 5 (best) Likert scale, measuring either the frequency of occurrence (always, most of the time, sometimes, rarely and never) or the degree of agreement with the item (completely agree, moderately agree, neither agree nor disagree, moderately disagree and completely disagree). As suggested by the authors of the questionnaire (11), a global score and scores of the respective scales and subscales were obtained by adding the results using the following formula:

$(\mathrm{X}-\mathrm{Y}) \div(5 \mathrm{Y}-\mathrm{Y}) 100$

where $X$ is the sum of the answers (between 1 and 5 for each item) of the respective scale and $Y$ is the number of items of the respective scale (22 for the total ACROQOLscore, 8 for the physical scale, 14 for the psychological scale, 7 for the subscales 'appearance' and 'personal relations'). The formula converts the different scores to a scale between a minimum of 0 (worst) and a maximum of 100 (best).

\section{Statistical analysis}

Quantitative data are presented as mean \pm s.D. and qualitative data as percentages. Comparisons among groups were performed using Student's t-test for quantitative data and $\chi^{2}$-test for qualitative variables. Both Student's $t$-test and multivariate linear regression analyses were performed to assess independent association of whether current lanreotide treatment or not with the ACROQOL scores of total score, respective scales, and subscales. All statistical methods were carried out with SPSS 12.0 for Windows software (SPSS Inc., Chicago, IL, USA). A $P$ value $<0.05$ was considered statistically significant.

\section{Results}

The cohort consisted of 52 patients with treated acromegaly. Half of the patients were male. The mean age was $51.9 \pm 10.1$ (s.D.) years, range 20-76 years. Mean time interval between diagnosis and study assessment was 12.6 \pm 7.1 years, range $1-30$ years. At diagnosis, 35 patients $(67.3 \%)$ had a macroadenoma and $17(32.7 \%)$ had a microadenoma. Out of these 52 patients, 16 (six male, ten female) were on hormone replacement because of surgery-related hypopituitarism. Of these 16 patients, 11 were on thyroxine replacement, 15 on glucocorticoids replacement, and 2 
males and 4 females were on sex steroid replacement. Out of ten females, two were menopausal and free of postmenopausal symptoms, who did not accept sex steroid replacement.

The clinical characteristics of the biochemically controlled and uncontrolled groups are shown in Table 1. Out of all 52 patients, $30(57.7 \%)$ were biochemically categorized as the controlled group and $22(42.3 \%)$ were categorized as the uncontrolled group. There was significant difference between groups in operation history, latest GH and IGF-I level $(P=0.042$, $<0.001,<0.001$ respectively). There was no significant difference between groups in age, gender, age of definite diagnosis, repeated operation history, radiotherapy history, percentage of macroadenoma, percentage of on hormone replacement, latest GH/IGF-I level and rate of morbidity related to acromegaly.

Distribution of the ACROQOL scores of total score, scales and subscales in controlled and uncontrolled groups is shown in Table 2. There was no significant difference between groups in the ACROQOL scores of total score, scales and subscales.

The biochemically controlled and uncontrolled groups were further divided into four groups according to present with or without lanreotide treatment. The clinical characteristics of these four groups are shown in Table 3. There were 13 patients $(25 \%)$ in the "controlled with present lanreotide treatment' group, 17 (32.7\%) in the 'controlled without present lanreotide treatment' group, $11(21.2 \%)$ in the 'uncontrolled with present lanreotide treatment' group and $11(21.2 \%)$ in the 'uncontrolled without present lanreotide' group. There was no significant difference between groups in age, gender, age of definite diagnosis, operation history rate, repeated operation history rate, radiotherapy rate, macro- or microadenoma, latest IGF-I level or morbidity rate. There was a significant difference between the 'controlled with present lanreotide treatment' and

Table 1 Clinical characteristics of biochemically controlled and uncontrolled groups.

\begin{tabular}{lllc}
\hline Variables & $\begin{array}{c}\text { Controlled } \\
(n=30)\end{array}$ & $\begin{array}{c}\text { Uncontrolled } \\
(n=22)\end{array}$ & $\boldsymbol{P}$ \\
\hline Age (years) & $52.0 \pm 8.7$ & $52.0 \pm 12.1$ & 0.987 \\
Male (\%) & 40 & 63.6 & 0.092 \\
Age of diagnosis (years) & $38.7 \pm 8.8$ & $41.6 \pm 9.9$ & 0.285 \\
Disease duration (years) & $12.9 \pm 6.5$ & $10.0 \pm 7.3$ & 0.135 \\
Operated (\%) & 93.3 & 72.7 & $0.042^{*}$ \\
Re-operated (\%) & 33.3 & 0 & 0.217 \\
Radiotherapy (\%) & 64 & 13.6 & 0.105 \\
Macroadenoma (\%) & 64 & 75 & 0.476 \\
Lanreotide use (\%) & 43.3 & 50.0 & 0.643 \\
Hormone replacement (\%) & 43.3 & 18.2 & 0.092 \\
GH ( $\mu \mathrm{g} / \mathrm{l})$, & $0.9 \pm 0.6$ & $7.8 \pm 8.7$ & $<0.001^{*}$ \\
$\quad 1 \mu \mathrm{g} / \mathrm{l}=46.5 \mathrm{pmol} / 1$ & & & \\
IGF-I $(\mu \mathrm{g} / \mathrm{l})$, & $187.0 \pm 86.8$ & $403.2 \pm 169.6$ & $<0.001^{*}$ \\
$\quad 1 \mu \mathrm{g} / 1=0.13 \mathrm{nmol} / \mathrm{l}$ & & 40.9 & 0.861 \\
Morbidity $(\%)$ & 43.3 & 40.9 & \\
\hline
\end{tabular}

$\mathrm{GH}$, growth hormone; IGF-I, insulin-like growth factor-I. ${ }^{*} P<0.05$, by Student's $t$-test for quantitative data and $\chi^{2}$-test for qualitative variables. 'controlled without present lanreotide treatment' groups in the latest $\mathrm{GH}$ level $(P=0.021)$. There was also a significant difference between the "uncontrolled with present lanreotide treatment' and 'uncontrolled without present lanreotide treatment' groups in the age of definite diagnosis $(P=0.044)$.

Distribution of the ACROQOL scores of total score, scales and subscales in the four groups was shown in Table 4 . There was a significant difference between the 'controlled with present lanreotide treatment' and 'controlled without present lanreotide treatment' groups in total score $(P=0.021)$, psychological scale $(P=0.011)$, psychological subscale 'appearance' $(P=0.032)$ and 'personal relationships' $(P=0.010)$.

\section{Discussion}

Our study demonstrated that there was no significant difference between biochemically controlled and uncontrolled acromegaly in the ACROQOL scores of total score, physical scale, psychological scale and psychological subscales. In the biochemically controlled group, those presently treated with slow-release somatostatin analogues (lanreotide), in comparison with without, had significantly worse quality of life, including the total score, psychological scale, and psychological subscales 'appearance' and 'personal relationships'. On the contrary, in the biochemically uncontrolled group, there was no difference between whether current treatment with lanreotide or not in the ACROQOL scores of total score, both scales, and subscales.

Although the abundance of evidences has demonstrated that administration of somatostatin analogues ameliorates the abnormal biochemical profiles and the associated somatic impairments (15-19), whether these improvements translate into improved physical function remains largely unknown, and their impact on HR-QOL is still controversial (20). Before the development of disease-generated ACROQOL, only two studies assessed the impact of slow-release somatostatin analogues on quality of life in patients with acromegaly.

An open treatment study by Sonino et al. investigated the effects on quality of life of slow-release somatostatin analogue (lanreotide) in ten patients with active acromegaly. Hormone measurements and psychometric evaluation by means of self-rating scales were carried out at the baseline and after 1 and 2 months. Together with a significant decrease in GH and IGF-I, treatment with lanreotide significantly improved symptoms relating to psychological distress, well-being and social fears by employing the Symptom questionnaire, the Cognitive Scale of the screening List for Psychosocial Problems by Kellner and the Social Situation Questionnaire by Marks (21). Another small prospective study assessing various outcome measures including quality of life in nine patients with newly diagnosed acromegaly treated with 
Table 2 Distribution of the acromegaly quality of life questionnaire scores of total score, scales and subscales in biochemically controlled and uncontrolled groups.

\begin{tabular}{lcccc}
\hline Variables & $\begin{array}{c}\text { Controlled } \\
(n=30)\end{array}$ & $\begin{array}{c}\text { Uncontrolled } \\
(n=22)\end{array}$ & $\boldsymbol{P}$ & $\boldsymbol{P}^{\zeta}$ \\
\hline Scale 1 & $59.3 \pm 30.6$ & $48.7 \pm 31.8$ & 0.232 & 0.258 \\
Scale 2 & $59.5 \pm 27.4$ & $56.6 \pm 26.5$ & 0.699 & 0.752 \\
Subscale 2-1 & $48.5 \pm 27.8$ & $45.6 \pm 29.9$ & 0.727 & 0.740 \\
Subscale 2-2 & $70.6 \pm 30.6$ & $67.5 \pm 26.0$ & 0.706 & 0.794 \\
Total score & $59.4 \pm 26.9$ & $53.7 \pm 27.0$ & 0.453 & 0.497 \\
\hline
\end{tabular}

Comparisons between groups using univariate and multivariate regression analyses. $P^{\zeta}$ calculated by multivariate regression analysis after adjustmen for age and gender. Scale 1, physical scale; scale 2, psychological scale scale 2-1, psychological subscale 'appearance'; scale 2-2, psychological subscale 'personal relationships'.

octreotide-LAR for 6 months demonstrated significantly improvements in health perception and fatigue (22).

On the contrary, our study showed that treatment with slow-release somatostatin analogues (lanreotide) impairs quality of life, especially the psychological aspect. Furthermore, this association only occurred in the biochemical remission patients, not in the biochemically uncontrolled group. In contrast with the previous two studies, our study recruited a larger cohort to evaluate the effect in patients in remission status or not, and used a different measure of quality of life questionnaire, ACROQOL. ACROQOL is a diseasegenerated quality of life questionnaire and represents a reliable, construct valid and disease-specific tool for assessing HR-QOL in patients with acromegaly $(23,24)$. Besides the opposite results compared with the previous studies, the most interesting findings are that lanreoide treatment selectively impairs quality of life in patients with treated acromegaly in remission status, independent of age and gender. This may imply that the remission status achieved by lanreotide treatment would significantly worsen quality of life, especially the psychological aspect. This negative effect on quality of life in acromegalic patients may be expressed in patients in remission status. The acromegalic patients in biochemical remission may function relatively normal in daily life physically and their overall mortality rate may be similar to that of general population (4). However, we speculate that the requirement of having slow-release somatostatin analogue (lanreotide) injection every 2 weeks in hospital may give the patients more psychological distress, social fears and less sense of well-being. This kind of impairment may be exaggeratedly expressed in the patients in remission status.

There are two studies assessing quality of life in patients with controlled versus uncontrolled acromegaly using ACROQOL. Furthermore, both studies used the same remission criteria as ours. A prospective and cross-sectional study by Trepp et al. (23) shows the results on 33 patients with treated acromegaly completing the German version of the ACROQOL. Patients with biochemically uncontrolled acromegaly had significantly lower HR-QOL than patients with acromegaly in remission status.

Another cross-sectional study by Rowles et al. (13) on 80 patients with acromegaly demonstrated that there is no correlation between biochemical control and any measure of quality of life, including ACROQOL, Psychological General Well-Being Schedule, EuroQoL, and signs and symptoms score. Our study results also support the latter findings.

That acromegaly biochemically controlled by slowrelease somatostatin analogues (lanreotide) leads to worse quality of life, and no difference between controlled and uncontrolled groups in the ACROQOL scores, in our study seems counterintuitive and runs contrary to the plethora of published data and vast personal experience of multiple researchers in this field: there is no doubt that controlled patients as a group individually feel better. Two potential explanations are possible. First, the magnitude of biochemical activity in

Table 3 Clinical characteristics of the four groups.

\begin{tabular}{|c|c|c|c|c|c|c|}
\hline \multirow[b]{2}{*}{ Variables } & \multicolumn{3}{|c|}{ Controlled } & \multicolumn{3}{|c|}{ Uncontrolled } \\
\hline & $\begin{array}{l}\text { Lanreotide }(+) \\
\quad(n=13)\end{array}$ & $\begin{array}{l}\text { Lanreotide }(-) \\
\quad(n=17)\end{array}$ & $P$ & $\begin{array}{l}\text { Lanreotide }(+) \\
\quad(n=11)\end{array}$ & $\begin{array}{l}\text { Lanreotide }(-) \\
\quad(n=11)\end{array}$ & $P$ \\
\hline Age (years) & $50.8 \pm 10.2$ & $52.9 \pm 7.5$ & 0.507 & $48.9 \pm 12.7$ & $55.0 \pm 11.1$ & 0.246 \\
\hline Male (\%) & 38.5 & 41.2 & 0.880 & 54.5 & 72.7 & 0.375 \\
\hline Age of diagnosis (years) & $37.5 \pm 8.9$ & $40.4 \pm 9.2$ & 0.383 & $37.8 \pm 9.0$ & $46.2 \pm 9.3$ & $0.044^{*}$ \\
\hline Disease duration (years) & $13.3 \pm 5.7$ & $12.5 \pm 7.2$ & 0.751 & $11.1 \pm 8.2$ & $8.8 \pm 6.4$ & 0.478 \\
\hline Operated (\%) & $92.3^{-0}$ & 94.1 & 0.844 & $81.8^{--1}$ & 63.6 & 0.338 \\
\hline Re-operated (\%) & 7.7 & 5.9 & 0.844 & 0 & 0 & - \\
\hline Radiotherapy (\%) & 38.5 & 29.4 & 0.602 & 18.2 & 9.1 & 0.534 \\
\hline Macroadenoma (\%) & 69.2 & 58.8 & 0.558 & 90.9 & 54.5 & 0.056 \\
\hline Hormone replacement (\%) & 46.2 & 35.3 & 0.547 & 27.3 & 9.1 & 0.269 \\
\hline $\begin{array}{l}\mathrm{GH}(\mu \mathrm{g} /) \\
\quad 1 \mu \mathrm{g} / \mathrm{l}=46.5 \mathrm{pmol} / \mathrm{l}\end{array}$ & $1.2 \pm 0.5$ & $0.7 \pm 0.6$ & $0.021^{*}$ & $5.9 \pm 3.0$ & $9.7 \pm 11.9$ & 0.308 \\
\hline $\begin{array}{l}\text { IGF-I }(\mu \mathrm{g} /) \\
\quad 1 \mu \mathrm{g} / \mathrm{l}=0.13 \mathrm{nmol} / \mathrm{l}\end{array}$ & $204.7 \pm 70.6$ & $170.7 \pm 84.3$ & 0.251 & $367.0 \pm 153.2$ & $435.7 \pm 161.5$ & 0.318 \\
\hline Morbidity (\%) & 38.5 & 47.1 & 0.638 & 36.4 & 45.5 & 0.665 \\
\hline
\end{tabular}

GH, growth hormone; IGF-I, insulin-like growth factor-I. ${ }^{*} P<0.05$, by Student's $t$-test for quantitative data and $\chi^{2}$-test for qualitative variables. 
Table 4 Distribution of the acromegaly quality of life questionnaire scores of total score, scales and subscales in four groups.

\begin{tabular}{|c|c|c|c|c|c|c|c|c|}
\hline \multirow[b]{2}{*}{ Variables } & \multicolumn{4}{|c|}{ Controlled } & \multicolumn{4}{|c|}{ Uncontrolled } \\
\hline & $\begin{array}{l}\text { Lanreotide } \\
(+)(n=13)\end{array}$ & $\begin{array}{l}\text { Lanreotide } \\
(-)(n=17)\end{array}$ & $P$ & $P^{\zeta}$ & $\begin{array}{l}\text { Lanreotide } \\
(+)(n=11)\end{array}$ & $\begin{array}{l}\text { Lanreotide } \\
(-)(n=11)\end{array}$ & $P$ & $P^{\zeta}$ \\
\hline Scale 1 & $49.5 \pm 34.4$ & $66.7 \pm 25.9$ & 0.129 & 0.118 & $55.1 \pm 30.4$ & $42.3 \pm 33.2$ & 0.358 & 0.533 \\
\hline Scale 2 & $45.5 \pm 29.7$ & $70.3 \pm 20.3$ & $0.011^{*}$ & $0.011^{*}$ & $55.7 \pm 24.8$ & $56.4 \pm 29.4$ & 0.989 & 0.736 \\
\hline Scale 2-1 & $36.3 \pm 29.5$ & $57.8 \pm 23.2$ & $0.033^{*}$ & $0.032^{*}$ & $47.4 \pm 26.2$ & $43.8 \pm 34.5$ & 0.787 & 0.986 \\
\hline Scale 2-2 & $54.7 \pm 34.1$ & $82.8 \pm 21.4$ & $0.010^{*}$ & $0.010^{*}$ & $65.9 \pm 24.2$ & $69.2 \pm 28.7$ & 0.777 & 0.470 \\
\hline Total score & $46.9 \pm 29.8$ & $69.0 \pm 20.5$ & $0.023^{*}$ & $0.021^{*}$ & $56.1 \pm 26.4$ & $51.3 \pm 28.6$ & 0.690 & 0.956 \\
\hline
\end{tabular}

Comparisons between groups using univariate and multivariate regression analyses. $P^{\zeta}$ calculated by a multivariate regression analysis after adjustment for age and gender. ${ }^{\star} P$ value $<0.05$. Scale 1 , physical scale; scale 2 , psychological scale; scale $2-1$, psychological subscale 'appearance'; scale $2-2$, psychological subscale 'personal relationships'.

uncontrolled patients was so mild (see Table 1), for example, a cross-sectional study by Damjanovic et al. (25) on 41 patients after transsphenoidal pituitary surgery for somatotroph pituitary adenoma showed that those achieving complete biochemical cure and those with discordant GH and IGF-I levels had similar beneficial changes in cardiac performance, insulin resistance and body composition. It appears that even incomplete disease control may not necessarily result in a clinically relevant unfavourable outcome if the disease activity is mild. Second, the ACROQOL may not be a good or a sensitive enough instrument, or the Chinese version was not equivalent.

Until now there has been a paucity of data attempting to correlate biochemical control and slow-release somatostatin analogues with quality of life in acromegaly. Furthermore, our study and previously published studies showed inconsistent results and the impact remained controversial. As with previous studies, the major limitation is the limited sample size. Thus, further investigations on this issue are mandatory. The possibly unknown psychological side effects caused by slowrelease somatostatin analogues should be further examined and evaluated. The negative impact on the quality of life, especially the psychological aspect, should be taken into serious consideration and psychotherapy may be needed in this field.

\section{References}

1 Melmed S. Acromegaly. New England Journal of Medicine 1990322 966-977.

2 Etxabe J, Gaztambide S, Latorre P \& Vazquez JA. Acromegaly: an epidemiological study. Journal of Endocrinological Investigation 1993 16 181-187.

3 Alexander L, Appletton D, Hall R, Ross WM \& Wilkinson R. Epidemiology of acromegaly in the Newcastle region. Clinical Endocrinology 198012 71-79.

4 Orme SM, McNally RJ, Cartwright RA \& Belchetz PE. Mortality and cancer incidence in acromegaly: a retrospective cohort study. United Kingdom Acromegaly Study Group. Journal of Clinical Endocrinology and Metabolism $1998 \mathbf{8 3} 2730-2734$.

5 Swearingen B, Barker FG, II, Katznelson L, Biller BM, Grinspoon S, Klibanski A, Moayeri N, Black PM \& Zervas NT. Long-term mortality after transsphenoidal surgery and adjunctive therapy for acromegaly. Journal of Clinical Endocrinology and Metabolism 1998 83 3419-3426.

6 Wright AD, Hill DM, Lowy C \& Fraser TR. Mortality in acromegaly. Quarterly Journal of Medicine 197039 1-16.

7 Bates AS, Vant Hoff W, Jones JM \& Clayton RN. An audit of outcome of treatment in acromegaly. Quarterly Journal of Medicine $199386293-299$.

8 Abosch A, Tyrrell JB, Lamborn KR, Haneegan LT, Applebury CB \& Wilson CB. Transsphenoidal microsurgery for growth hormonesecreting pituitary adenomas: initial outcomes and long-term results. Journal of Clinical Endocrinology and Metabolism $1998 \mathbf{8 3}$ $3411-3418$

9 Giustina A, Barkan A, Casanueva FF, Cavagnini F, Frohman L, Ho K, Veldhuis J, Wass J, Von Werder K \& Melmed S. Criteria for cure of acromegaly: a consensus statement. Journal of Clinical Endocrinology and Metabolism 200085 526-529.

10 Webb SM, Prieto L, Badia X, Albareda M, Catalá M, Gaztambide S, Lucas T, Páramo C, Picó A, Lucas A, Halperin I, Obiols G \& Astorga R. Acromegaly quality of life questionnaire (ACROQOL), a new health-related quality of life questionnaire for patients with acromegaly: development and psychometric properties. Clinical Endocrinology 200257 251-258.

11 Badia X, Webb SM, Prieto L \& Lara N. Acromegaly quality of life questionnaire (AcroQoL). Health and Quality of Life Outcomes 2004 27 2-13.

12 Webb SM, Páramo C, Astorga R, Picó A, Gómez JM, de Pablos P, Benito P, Varela C, Lucas-Morante T, Moreno B, Catalá M, Gaztambide S, Halperin I, Torres E, Lucas A, Badia X \& Gilabert M. Longitudinal study of health-related quality of life (HR-QoL) in acromegaly, evaluated with the Acroqol Questionnaire 11th Meeting of the European Neuroendocrine Association ENEA Oral Communication, Munich, Germany 2004.

13 Rowles SV, Prieto L, Badia X, Shalet SM, Webb SM \& Trainer PJ. Quality of life (QOL) in patients with acromegaly is severely impaired: use of a novel measure of QOL: acromegaly quality of life questionnaire. Journal of Clinical Endocrinology and Metabolism $2005903337-3341$.

14 Herdman M, Fox-Rushby J \& Badia X. A model of equivalence in the cultural adaptation of HRQoL instruments: the universalist approach. Quality of Life Research 19987 323-335.

15 Ezzat S, Snyder PJ, Young WF, Boyajy LD, Newman C, Klibanski A, Molitch ME, Boyd AE, Sheeler L, Cook DM, Malarkey WB, Jackson I, Vance ML, Thorner MO, Barkan A, Frohman LA \& Melmed S. Octreotide treatment of acromegaly. A randomized, multicenter study. Annals of Internal Medicine 1992117 711-718.

16 Flogstad AK, Halse J, Haldorsen T, Lancranjan I, Marbach P, Bruns C \& Jervell J. Sandostatin LAR in acromegalic patients: a dose-range study. Journal of Clinical Endocrinology and Metabolism 199580 3601-3607.

17 Arosio M, Macchelli S, Rossi CM, Casati G, Biella O \& Faglia G. Effects of treatment with octreotide in acromegalic patients - a multicenter Italian study. Italian Multicenter Octreotide Study Group. European Journal of Endocrinology 1995133 430-439. 
18 Suliman M, Jenkins R, Ross R, Powell T, Battersby R \& Cullen DR. Long-term treatment of acromegaly with the somatostatin analogue SR-lanreotide. Journal of Endocrinological Investigation 199922 409-418.

19 Giusti M, Ciccarelli E, Dallabonzana D, Delitala G, Faglia G, Liuzzi A, Gussoni G \& Giordano DG. Clinical results of long-term slow-release lanreotide treatment of acromegaly. European Journal of Clinical Investigation 199727 277-284.

20 Woodhouse LJ, Mukherjee A, Shalet SM \& Ezzat S. The influence of growth hormone status on physical impairments, functional limitations, and health-related quality of life in adults. Endocrine Reviews 200627 287-317.

21 Sonino N, Scarpa E, Paoletta A, Fallo F \& Boscaro M. Slowreleasing lanreotide treatment in acromegaly: effects on quality of life. Psychotherapy and Psychosomatics 199968 165-167.

22 Gilbert J, Ketchen M, Kane P, Mason T, Baister E, Monaghan M, Barr S \& Harris PE. The treatment of de novo acromegalic patients with octreotide-LAR: efficacy, tolerability and cardiovascular effects. Pituitary 20036 11-18.
23 Trepp R, Everts R, Stettler C, Fischli S, Allemann S, Webb SM \& Christ ER. Assessment of quality of life in patients with uncontrolled vs controlled acromegaly using the Acromegaly Quality of Life Questionnaire (AcroQoL). Clinical Endocrinology 200563 103-110.

24 Rowles SV, Prieto L, Badia X, Shalet SM, Webb SM \& Trainer PJ. Quality of life (QOL) in patients with acromegaly is severely impaired: use of a novel measure of QOL: acromegaly quality of life questionnaire. Journal of Clinical Endocrinology and Metabolism $2005903337-3341$.

25 Damjanovic SS, Neskovic AN, Petakov MS, Popovic V, Macut D, Vukojevic P \& Joksimovic MM. Clinical indicators of biochemical remission in acromegaly: does incomplete disease control always mean therapeutic failure? Clinical Endocrinology $2005 \mathbf{6 2}$ 410-417.

Received 16 June 2006

Accepted 12 September 2006 